\title{
LOS CONTRATOS, LAS ASIMETRÍAS DE LA INFORMACIÓN EN SALUD, EL RIESGO MORAL Y LA SELECCIÓN ADVERSA
}

\section{RESUMEN}

$\boldsymbol{E}_{\text {ste }}$ articulo pretende plantear las situaciones en las que existe información asimétrica en la relación contractual en el Sistema General de Seguridad Social en Salud, es decir, en las que un actor sabe algo que el otro desconoce. Se tratan dos grandes tema: riesgo moral, selección adversa,etc. Con el propósito de contextualizar al lector sobre el problema que se plantea y particularizar las diferencias, con el tema contractual.

En los últimos quince años posiblemente la economía de la información es, dentro de la Teoría Económica, la que más ha evolucionado. Se fundamenta su objetivo en la búsqueda y estudio de las consecuencias de la existencia de asimetría de información entre diferentes agentes económicos, sobre su organización y operación.

\section{Palabras clave}

Asimetría de la información Riesgo moral

Selección adversa, contractual.
Eduardo Antonio Cano Plata* Carlos Alberto Cano Plata **

\section{InTRODUCCIón}

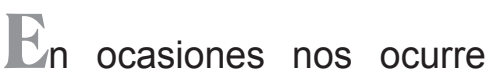
que al ir a una empresa, no nos prestan el servicio que requerimos. A las grandes filas se suma el hecho de que los trabajadores conversan tranquilamente, sin ningún tipo de preocupación, y hablan por teléfono con familiares o amigos sobre algunos temas irrelevantes. Así se podría generar el comentario de alguien que diga: "Se ve que lo que ganan no depende del número de usuarios que atienden"; en esas palabras se refleja una crítica a la falta de incentivos de los empleados, por lo tanto, este tipo de contratos muestra una deficiencia del sistema. (Macho, I \& Pérez, 1994).

\footnotetext{
* PhD.Universidad de Buenos Aires. Profesor asistente Universidad Nacional de Colombia.

** Administrador de Empresas Universidad Nacional de Colombia, Especialista en Derecho Empresarial UNAB, Magister en Administración Universidad Nacional de Manizales.
} 
Los contratos en el Sistema de Salud, particularmente los existentes entre las EPS (Entidad Promotora de Salud) o ARS (Administradora de Régimen Subsidiado en Salud) y los afiliados, en el que se ven involucrados los médicos y los centros asistenciales o IPS (Institución Prestadora de Servicios de Salud), presentaran unas características enmarcadas dentro del POS (Plan Obligatorio de Salud) y el PAB (Plan de Atención Básica), ligados al marco normativo que establece los procedimientos y requisitos para la prestación del servicio de salud, estos contratos son los que se van a analizar. (Cano, C .2004).

El cliente elige de acuerdo con su capacidad de pago bien sea, el régimen contributivo (EPS) o el régimen subsidiado (ARS). En estos contratos, cada vez que se presente un problema de salud, la compañía cubre los gastos y el paciente aporta en el régimen contributivo el $12.5 \%$ discriminados así: al patronal le corresponde el $8.5 \%$ y al trabajador en un $4 \%$ adicionalmente se debe aportar en el régimen contributivo, las cuotas moderadoras por los servicios recibidos, o el Copago por la prestación de servicios de los beneficiarios del portante. (Ley 1122 de 2007)

\section{Otra modalidad de contrato es} el de la ARS, sensiblemente más barata, por ser régimen subsidiado, donde en caso de enfermedad esta cubre sólo los gastos que no pueden sobrepasar una cuantía determinada, el resto es cubierto por el FOSYGA ${ }^{1}$ (Varela, A, 2002).

\section{ABstraCt}

This paper describes the situations in which there is evidence of asymmetrical information in the contractual relation in the Health Social Security System; that is to say, situations in which an agent knows something that another ignores. This paper deals with two main topics: Moral Risk and Adverse Selection and it aims at contextualizing the readers on the presented problem and making all differences clear, having the contractual issue as its principle focus.

In the last 15 years, information economy may be the most advanced within the economic theory. It is based on the objective of exploring and analyzing the existing information asymmetry among different economic agents, its organization and operation.

\section{KEY WORDS}

\author{
Information asymmetry \\ Moral risk \\ Adverse Selection \\ Contractual
}


Diferentes tipos de usuarios están interesados por diferentes coberturas, por lo que existen entonces, alternativas adicionales como la medicina prepago, que en algunos casos son ofrecidos por las mismas EPS contemplando diferentes alternativas, con cubrimientos distintos de acuerdo con la capacidad de pago del que toma el contrato.

En otros casos la contratación tiene, sin embargo, propiedades distintas. Se propone el acuerdo sólo a quien cumple ciertas características; es usual encontrar en la selección de demandas de trabajo de un periódico anuncios como la siguiente:

"Empresa busca joven profesional recién egresado", sin especificar el tipo de estudios realizados. No deja de ser extraño que solicite haber seguido estudios universitarios sin especificar cuales, sobre todo porque no parece que el solo hecho de haber pasado por la universidad dé ninguna capacitación para un trabajo concreto (caso típico en las empresas del estado). (Castaño, G. 2001).

\section{Astmetría y ACtores}

\begin{abstract}
Consideremos una relación bilateral $^{2}$ en la que una parte contrata a la otra para que le preste los servicios asistenciales que éste requiere; en el momento de asistir a su consultorio o al centro asistencial donde prestan los servicios y le permita así, tomar ciertas decisiones. A la parte contratante ${ }^{3}$ la llamaremos a partir de ahora, el principal, mientras que nos referiremos a la parte
\end{abstract}

contratada 4 como agente. Tanto el principal como el agente pueden referirse a personas, pero también se puede tratar de instituciones, organizaciones u otros centros de decisión. Podemos tomar como ejemplo la relación entre comprador de vehículos (el principal) y el equipo de vendedores de ésta (el agente). (Macho, I\&Pérez, 1994), (Stiglitz E and Welss A., 1981).

\footnotetext{
${ }^{1}$ Fondo de Solidaridad y Garantías en Salud adscrito al Ministerio de Protección Social.

2 Paciente-médico o médico.IPS.

${ }^{3}$ Paciente o médico

${ }^{4}$ Médico, EPS, ARS o IPS.
} 
El objetivo del contrato es que el agente realice una acción que beneficie al principal. (Cano, C, 2004). En el ejemplo de salud, el equipo de médicos es contratado para atender a los usuarios de la IPS. El contrato firmado entre ambas partes especificará los pagos que el principal efectuará al agente. Vamos a suponer que quien diseña el contrato siempre es el principal y que tras hacerlo, se lo ofrece al agente. Este, después de estudiar los términos del contrato, decide si acepta o no en relación con el principal; el agente acepta la propuesta del principal cuando la utilidad que tiene en este caso, es más alta a la que se puede garantizar si no firma el contrato. Se llamará utilidad de reserva a dicho nivel de utilidad. No es permisible, por tanto, que el agente haga contraofertas al principal, lo cual desencadenaría un proceso de negociación.

Se están considerando aquellas situaciones en las que el principal tiene todo el poder de negociación. Él decide los términos del eventual acuerdo, mientras que la decisión del agente se refiere sólo a si interviene o no. El principal, al ofrecer un contrato al agente, le dice: "Esto es lo que propongo; o lo toma o lo deja”. (Macho, I \& Pérez, 1994)

Si el agente decide tomar la decisión de no firmar, entonces el acuerdo no tiene lugar y no habrá ningún problema. Si, de lo contrario, acepta la propuesta, entonces debe efectuar, a la vista de los términos del convenio, las acciones para las que ha sido contratado. En el ejemplo de la relación entre vendedores de vehículos y compradores, el equipo de ventas debe decidir la estrategia de la empresa, y esto supone dedicar esfuerzos a distintas tareas y proyectos para conseguir su objetivo final (vender vehículos). (Akerlof G, 1970), (Stiglitz E and WelssA., 1981).

Hasta el momento, los comportamientos estratégicos básicos en la relación de esta situación son los siguientes:

- El principal (EPS, IPS, ARS) diseña el contrato, o el conjunto de contratos, que ofrecerá el agente (médico).

- El agente acepta el contrato si ello le conviene, es decir, si este le garantiza una utilidad esperada superior a las otras oportunidades quetiene abiertas.

- El agente realiza una acción o esfuerzo para el principal. (Macho, I \& Pérez, 1994), (Varela, A, 2002)

\section{De estos comportamientos} se deduce que los objetivos del agente están en conflicto con los del principal. Lo que para uno supone un costo, para otro es un ingreso. El valor pagado es un ingreso para el agente, pero un costo para el principal; el esfuerzo del agente beneficia al principal, pero es costoso para este. 


\begin{abstract}
A continuación se expone lo que se entiende por un contrato: es un acuerdo de voluntades para ambas partes, en el que se especifican las obligaciones de una y el deber de la otra, con el propósito de cubrir una labor específica o cualquier contingencia derivada de la relación contractual. En particular incluye el mecanismo de pago con el que remunerará al agente.
\end{abstract}

Un punto a tener en cuenta es que un contrato sólo pude estar basado en variables verificables. En otras palabras, sus términos sólo pueden depender de variables comprobables por agentes externos a la relación, ya que garantiza su cumplimiento. Utilizar variables verificables en el contrato permite a cualquiera de las partes acudir a un juez con "pruebas" sobre lo acontecido y reclamar que los términos del acuerdo se cumplan.

¿Qué ocurre con un contrato cuya información no puedeserverificada?, en esta circunstancia es difícil acudir a un tercero para que interceda en caso de desacuerdo; por ejemplo a un juzgado, ya que éste no tendrá elementos de juicio para comprobar, si efectivamente lo que están reclamando las partes, es cierto o no ${ }^{1}$. Por lo tanto, como todos los participantes saben que la intermediación es imposible, tendrá interés en violar los términos del acuerdo, anticipando este comportamiento. Ninguno deseará firma un contrato que luego no se va a respetar y sobre el que no se puede reclamar indemnización alguna por incumplimiento (factor ético en el caso de salud). (Cano C, 2004), (Castaño R, 1999).

\section{La información}

Ja información está relacionada con el conjunto de variables que son verificables en una relación. Para entender cómo entra este aspecto en juego, volvamos al caso de la relación entre la IPS y el médico que contratan. La relación se establece para que este último mantenga en buen estado de salud a los pacientes. Es evidente que los pacientes preferirán el mejor equipo de médicos, aunque resulte algo más caro. Sin embargo, parece claro que las IPS no pueden evaluar ni controlar perfectamente las decisiones de los médicos frente al

\footnotetext{
1 Esta situación es muy frecuente en el sistema de salud, por tratarse de un sistema altamente borroso y en el cual, solo se tiene acceso a la información relacionada con el estado real del paciente, mediante la historia clínica, a la que únicamente tienen acceso los médicos o en caso excepcionales, por orden judicial los jueces.
} 
estado real de salud de sus pacientes; ello imposibilita que el contrato se base en el comportamiento de los gestores, ya que este es intrínsecamente no verificable. Además, los pacientes no tienen información perfecta sobre las características del equipo que están formando tanto a nivel individual como a nivel de comportamiento del grupo. Por tanto, será también difícil establecer contratos en función de ciertos niveles de competencia o de calidad de los agentes. Estas ventajas informativas de los equipos de las EPS o ARS les dan cierto margen de maniobra para perseguir su propio provecho y no el de los pacientes.

Con las anteriores descripciones se observan las situaciones en las que en una relación contractual existe información asimétrica, es decir, en las que un participante sabe algo que el otro desconoce. Se analizan relaciones entre dos individuos o instituciones en la que uno de los participantes tiene o tendrá una ventaja informativa, sobre el otro individuo y los objetivos de ambos están en conflicto. La razón de mezclar asimetría de información y conflicto es que si los intereses del principal y del agente coinciden, la transmisión de toda la información relevante sería automática, y no habría asimetría. (Castaño, G. 2001).

"La teoría está dividida en tres grandes temas: riesgo moral, selección adversa, y señalización. En las situaciones de riesgo moral el principal no puede controlar el comportamiento del agente. En este caso, la solución pasa por trasmitirle incentivos a través del contrato. Una situación de selección adversa surge cuando el agente conoce antes de firmar el contrato un elemento relevante de la relación que el principal desconoce. La solución a este problema sugiere ofrecer distintas alternativas contractuales que lleven al agente a revelar su información. Finalmente, los modelos de señalización están relacionados con la situación en la que una de las partes conoce alguna variable importante para la relación y con su comportamiento puede señalar al otro participante."

\subsection{Relación temporal de los participantes}

Se supondrá en este modelo que la información con la que cuenta el principal y el agente en todo momento es la misma. Es decir, el principal y el agente se conocen ${ }^{1}$ y el esfuerzo del agente es una variable verificable y por lo tanto es posible comprobar cómo cumple con su tarea. Más adelante esto permitirá determinar cuáles son las características de la relación y la asimetría existente entre la información del principal y el agente. En este caso, por ejemplo, el principal y el agente no tienen dudas sobre quién firma el contrato; el principal ofrecerá a cada tipo de agente un contrato distinto ${ }^{2}$.

Del mismo modo, el agente aceptará distintos contratos según el principal que le esté contratando, y el

\footnotetext{
1 Cada participante conoce las características de su interlocutor, que son pertinentes para la relación contractual.

2 En función de sus capacidades, conocimientos, comportamientos, etc.
} 
trabajo o servicio para el que le contrate; ya que también se puede comprobar cuánto esfuerzo dedica el agente en la relación, es posible introducir esta variable en el contrato, además del resultado. Es preciso señalar que no se supone que hay conocimiento perfecto en todos los aspectos de la relación, sino que ambos participantes saben lo mismo. Por ello se dirá que las situaciones de referencia tienen información asimétrica. Esto no excluye que pueda haber elementos de carácter borroso ${ }^{1}$, para representar la relación de una variable borrosa se dirá, que la naturaleza decide (Giles D.E., \& Draeseke R. 2001)

FIGURA 1

Contrato

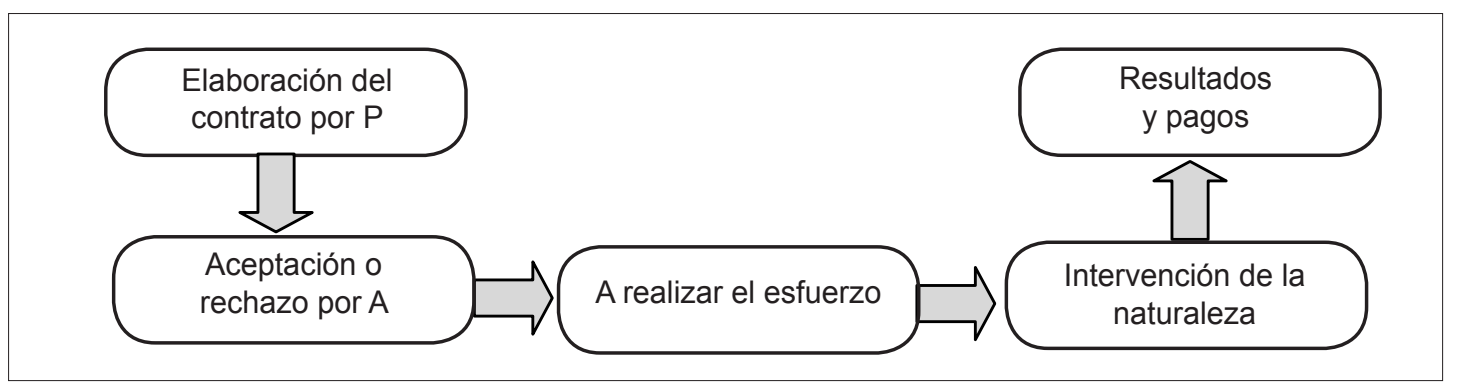

Fuente. Autor

El esquema temporal de referencia está resumido en la figura1, donde $\mathrm{P}$ es el principal y el agente. Este esquema pone en evidencia la secuencia de los participantes en la toma de las decisiones. Esta secuencialidad indica ya el tipo de concepto de solución que se necesita aplicar, - el de Cúmulo Borroso-. Este criterio de solución exige que todo instante de tiempo cada participante elija la estrategia más adecuada, dada la situación a la que se ha llegado y suponiendo que los demás participantes harán otro tanto. Es decir, el agente realizará el esfuerzo que maximiza su utilidad dado que ha aceptado el contrato ofrecido por el principal; anteriormente, y anticipando su decisión de esfuerzo, decide aceptar o no el contrato: el primero en actuar es el principal, que calcula el comportamiento futuro del agente para cada contrato posible y ofrece aquel que maximice su utilidad². (Giles D.E., \& Draeseke R.2001)

Es fácil aceptar que en las relaciones de tipo contractual siempre existen razones de peso por las cuales una parte posee más información que la otra sobre le objeto del contrato. Sin embargo, en distintas situaciones surgen formas de asimetría de información diferentes. Al distinguir las diferentes asimetrías que se presentan permite identificar la influencia de la naturaleza de la información sobre los aspectos del contrato. El intuir las expresiones lingüísticas en las relaciones contractuales lo hace asimétrico y borroso. (Cano C.2006), (Giles D.E., \& Draeseke R.2001)

\footnotetext{
1 La lógica borrosa se recomienda para soluciones de tipo lingüístico en los procesos que involucran variables no verificables.

2 La función objetivo a maximizar desde el punto de vista del cúmulo depende de la matriz de decisión del grado de borrosidad de los datos, y el algoritmo de clasificación que se emplee.
} 


\section{Problemas de información asimítirica}

\subsection{Riesgo moral}

El problema de riesgo moral se presenta cuando el agente recibe información privada una vez inicia la relación contractual ${ }^{1}$, o cuando la acción del agente no es verificable ${ }^{2}$. En las situaciones con riesgo moral los participantes disponen de la misma información, en el momento de firmar el contrato. La información asimétrica se deriva del hecho de que una vez establecido el vínculo, el principal no puede verificar o no puede observar la acción o el esfuerzo que el agente realiza, o no tiene mecanismos que le permitan controlar perfectamente esta acción ${ }^{3}$; esta característica evalúa un comportamiento estratégico de los actores que depende del estado de haber firmado ya un contrato y que hace del agente el manejo de variables altamente borrosas y de difícil verificación para la ejecución del contrato. (Akerlof G, 1970), (Spence M, 2001), (Castaño R, 1999)

Una forma de modelar este tipo de situación, es suponer que el esfuerzo del agente, que se realiza después de firmado el contrato, no es verificable, y en consecuencia esta variable no puede ser incluida en el contrato ${ }^{4}$. Por lo tanto, el pago del agente no puede depender del conocimiento que incorpora y para el que se le ha contratado. En la figura 2 se muestra este esfuerzo

FIGURA 2

Comportamiento estratégico basado en riesgo moral

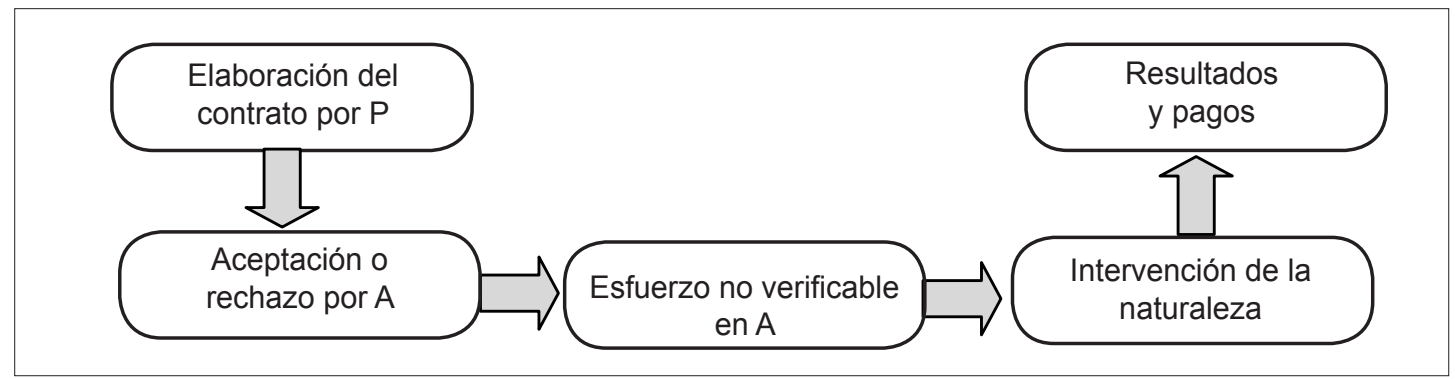

Fuente. Autor

\footnotetext{
${ }^{1}$ Información de otras EPS o ARS de las diferentes historias clínicas que reposan en sus archivos.

2 En el caso particular el médico.

${ }^{3}$ Caso típico en el sistema asistencial de salud.

${ }^{4}$ Para el caso de salud por tratarse de un sistema altamente borroso el contrato está sujeto a estas variables no verificables.
} 
Es fácil imaginar situaciones laborales en las que a pesar de que el resultado del esfuerzo del médico (el agente) sea verificable, como puede ser el caso cuando se trata de un número de pacientes atendidos en un día, su esfuerzo no es verificable para el IPS (el principal). Este es el caso de una Empresa Promotora de Salud que contrata a un médico para que atienda a domicilio los pacientes que ella tiene adscritos. El médico aceptará el contrato que le ofrecen si este contrato le garantiza al menos la misma utilidad esperada que las otras oportunidades abiertas en el mercado. El resultado en este caso es el nivel de pacientes atendidos. Es fácil aceptar que el número de pacientes atendidos por el agente es una variable verificable. Sin embargo, el esfuerzo del médico es extremadamente difícil de medir; de hecho el tiempo dedicado a cada paciente es incontrolable. Por lo tanto, la IPS no puede hacer depender el pago del agente directamente del esfuerzo de este.
Este tipo de problemas obliga a replantearse en algunos casos el enfoque tradicional en el que el trabajo ${ }^{1}$, se intercambia por un salario fijo, esto nos muestra el alto grado de borrosidad del sistema asistencial. (Giles D.E., \& DraesekeR.2001)

Sin embargo, no todos los problemas de Riesgo Moral se encuentran representados en la gráfica anterior. Algunos problemas englobados dentro de esta categoría se plantean cuando la diferencia de información se debe a que antes de realizar el esfuerzo para el que ha contratado, el agente observa una reacción de la naturaleza que no observa el principal ${ }^{2}$. Esto quiere decir que, la incertidumbre es la misma para ambos participantes cuando el contrato se firma. Sin embargo, antes de su ejecución, el agente tendrá una ventaja informativa sobre una variable que es importante para la relación, esto lo pone en una condición favorable en el comportamiento estratégico del contrato a establecer, reflejado en la figura 3. (Cano C, 2006), (Giles D.E., \& Draeseke R. 2001.

FIGURA 3

Nivel óptimo de esfuerzo del agente

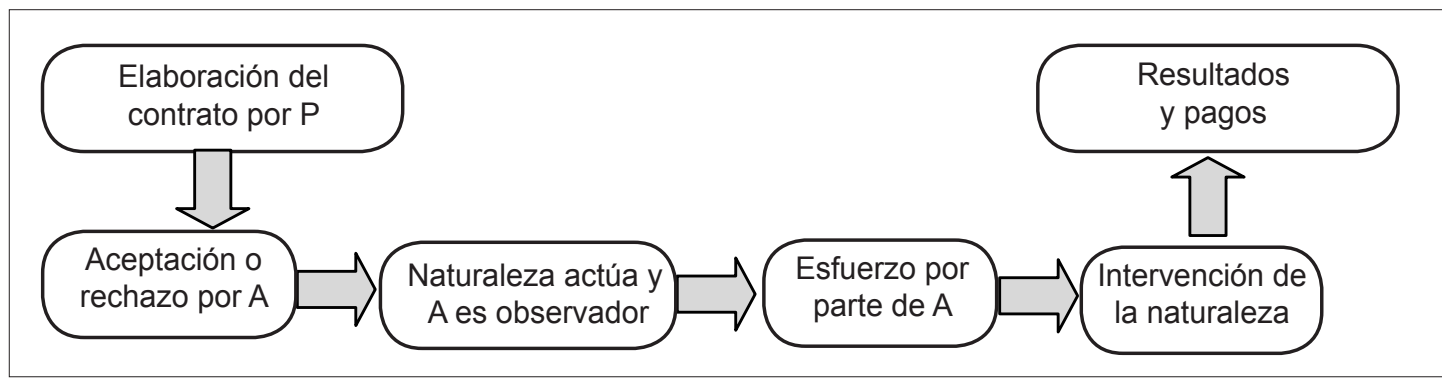

Fuente. Autor

\footnotetext{
1 Medido en número de horas.

2 Inferencias lingüísticas de tipo borroso.
} 
Este desarrollo corresponde, por ejemplo, a una EPS que contrata a un médico para que atienda pacientes dentro y fuera de la IPS. En algunos casos, la empresa tiene poca información sobre otros servicios existentes en las otras EPS, el estado de los mercados, etc., que influye en la mejor estrategia para prestar un mejor servicio domiciliario. Sin embargo, a pesar de que el agente no conozca estos datos cuando firma el contrato, los aprende cuando se ocupa de prestar el servicio. El principal puede conoce la estrategia que su agente ha adoptado, para él es difícil evaluar si la actuación del agente respecto a la atención fue la más pertinente dadas las características del mercado o no.

\subsection{Selección adversa}

Cuando el agente dispone de información que no posee el principal, antes del inicio de la relación se puede decir entonces que hay selección adversa. En este caso, el principal puede verificar el comportamiento del agente dentro de la relación; sin embargo, la decisión óptima o el costo de ésta, depende del tipo del agente o de ciertas características de la producción que solo conoce el agente (Vera, A. 2001).

Cuando las características del agente son las que hacen parte de la asimetría de la información, el principal sabe que el agente puede ser de varios tipos, pero no puede identificarlos ${ }^{1}$. Esta situación puede ser modelada suponiendo que la naturaleza selecciona primero el tipo de agente utilizando un clasificador difuso (figura 5.3). Se trata entonces de un cúmulo con información asimétrica previa a la firma del contrato, lo que se podría denominar, un cúmulo borroso con conocimiento "a priori". (Giles D.E., \& Draeseke R.2001)

FIGURA 4

Comportamiento estratégico basado en selección adversa

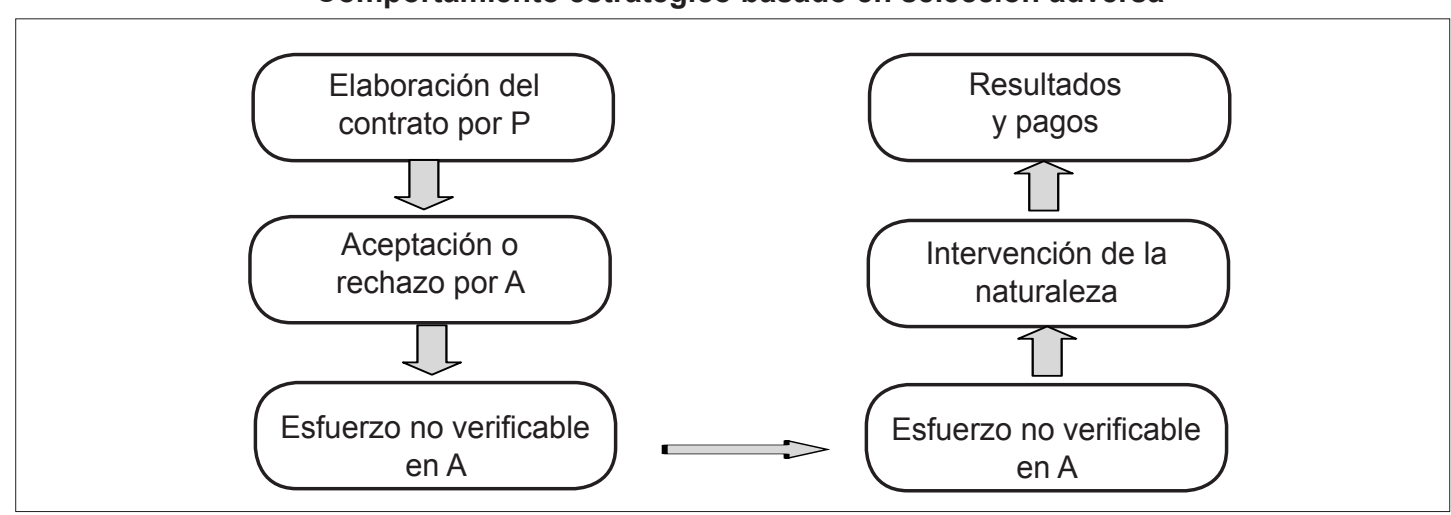

Fuente. Autor

1 Frecuente en el sistema de salud. 
No es fácil que una EPS pueda identificar a qué tipo de individuo pertenece un cliente cuando éste acude a contratar un seguro. En los seguros de salud, no es lo mismo que el cliente sea alguien muy cuidadoso en general o que, por el contrario, sea un descuidado con su salud, el que siempre se está enfermando. A la EPS o ARS le gustaría tener esta información para establecer contratos distintos en cada caso, con el fin de proponer programas de promoción y prevención adecuados y disminuir los costos que implica el sistema curativo en salud. Pero cuando un individuo acude a una EPS o ARS para afiliarse, los factores que inciden en la toma de decisiones de él ${ }^{1}$, no son conocidas por la empresa y lo que el individuo le diga espontáneamente no le parecerá muy fiable generando en consecuencia un sistema borroso para el análisis. Por lo tanto, estamos ante una situación como la que refleja la figura 5.3. (Vera, A. 2001).

Estos aspectos son los comportamientos estratégicos que los actores en la relación contractual tienen.

Además, el Sistema General de Seguridad Social presenta variables exógenas y endógenas asociadas con el crecimiento acelerado de los costos de la atención en salud, así como los efectos derivados de políticas públicas, (variable netamente exógena), que son abordados por la literatura internacional bajo estos criterios:
- La transición de la salud (estado de salud), variable altamente borrosa que consiste en que el perfil epidemiológico de un país cambia como resultado de una variación en el perfil demográfico y de una mejoría en la calidad de vida de las personas. Como resultado de ello, las enfermedades crónicas y degenerativas empiezan a tener mayor importancia sobre la carga de la enfermedad, en comparación con las enfermedades infecto -contagiosas e inmunoprevenibles. (Varela, A, 2002)

- Los cambios en los precios relativos entre los bienes y los servicios (Estatus socio económico). La producción de bienes de consumo se diferencia de la producción de servicios, entre muchas otras cosas, porque la segunda es intensiva en mano de obra, mientras que la primera es intensiva en capital (Equipos). Según esto, es de esperarse que los avances de la tecnología produzcan reducciones más drásticas en los costos de producción de bienes que en los costos de producción de servicios; esto hace que los productos sean cada vez más baratos por que se inventan nuevos procesos que ahorran más insumos, o que permiten lograr economías de escala importantes, tal como sucede por ejemplo en la producción de vehículos. (Roach, SS 1991).

1 Estado de salud, demografía, estrato socio económico, sexo, hábitos de vida, fumador, sobrepeso, bebe habitualmente alcohol o no, etc. 
En oposición al sector de bienes de consumo, el sector de servicios no se ha beneficiado tanto de los avances tecnológicos en los procesos de producción, puesto que la parte más importante del costo es la mano de obra. En el sector salud esto es tal vez más crítico: por mucho que se automaticen los procesos de producción, sigue siendo necesario que el médico examine a su paciente y haga el ejercicio del diagnóstico y la toma de decisiones clínica para cada caso individual; ni siquiera un cirujano cuya producción se puede maximizar delegando parte del proceso a auxiliares o a médicos en entrenamiento, podrá incrementar su productividad por encima de cierto nivel máximo. Por esta razón no se podrán lograr disminuciones importantes en los costos de la tecnología al menos hasta hoy, no se puede obviar la necesidad de que los servicios de salud se tengan que prestar de manera personal e individual, dificultando el acceso de ciertos sectores poblacionales (riesgo moral vs. selección adversa). (Roach, SS1991).
- La presencia de seguros o terceros pagadores (Cubrimiento de seguro individual). Este tópico se comenta desde el fenómeno de riesgo moral y su efecto generador de ineficiencia distributiva, así como la presencia de seguros facilita la inducción de la demanda por parte del profesional. Esto quiere decir que la presencia de un segundo o tercer pagador hace que el consumidor se vuelva menos sensible al precio del servicio de salud a medida que va disminuyendo el nivel de copago. En otras palabras entre menos tenga que pagar por el servicio se tendiende a consumir más. Esto es aplicable tanto para el régimen contributivo como para el régimen subsidiado. Es de aclarar que el efecto del riesgo moral es más intenso en aquellos servicios cuya demanda es elástica; lo cual es de esperarse, puesto que se trata de enfermedades que dan espera, es decir, que el paciente puede soportarlas mientras no sean problemas severos. (Alman SH, 1990). 


\section{Conclustones}

En este ensayo se trata el problema contractual, en la Economía de la Información se centran varios aspectos fundamentales en las relaciones contractuales. Una de ellas es la consecuencia que uno de los participantes tendrá sobre la ventaja informativa del otro participante, además de esta característica, sólo son interesantes aquellas situaciones en las que los objetivos de los participantes están en conflicto.

Se deduce de los comportamientos estratégicos en la relación contractual que los objetivos del agente están en conflicto con los del principal. Lo que para uno supone un costo, para otro es un ingreso. El valor pagado es un ingreso para el agente, pero un costo para el principal; el esfuerzo del agente beneficia al principal, pero es costoso para el agente.

En función de cuándo se origina la asimetría de información, aparecen diferentes tipo de modelos cada modelo genera comportamientos estratégicos distintos. Hay un problema de riesgo moral cuando la información asimétrica se produce tras la firma del contrato. Se dirá que existe un problema de selección adversa cuando antes de la firma el agente dispone de más información que el principal.

\section{CAMBLAMOS! \\ Correos \\ de Colombia \\ $\longrightarrow=$ \\ ADPOSTAL \\ Pensando en ofrecerle el mejor servicio \\ Nuestras Líneas de Atención al Cliente \\ $4298487-2633484-2956896$ \\ $018000111210 / 111313$ \\ Fax: 4163026 \\ Subgerencia de Mercadeo 3340304 \\ División de Mercadeo Regional DC 4297320




\section{Bibliografía}

Akerlof G A, (1970). The market for "Lemons" quality uncertainty and the market mechanism" Department of Economics, University of California, Berkeley, USA.

Akerlof. G. A, (2001). Behavioral Macroeconomics And Macroeconomic Behavior Prize Lecture, Decembers, * Department Of Economics, University Of California, Berkeley, USA

Altman SH, Goldberger S, Grane SC. (1990) The Need for a National Focus on Health Care Productivity Health Affairs 1990; 9 (1): pp, 107 -113.

Cano C, (2004). La salud como un problema de asimetría de la información caso del sector Público' sector privado. Decisiones administrativas. Universidad Nacional de Colombia: V.8, P.7 17, 2004.

Cano C (2006). Microeconometría y análisis de la demanda asistencia! al servicio de salud en el Eje Cafetero, factores explicativos de la decisión del paciente.: un caso de asimetría de la información. Tesis de grado Maestría en Ciencias de la Administración Universidad Nacional de Colombia P 29-40.

Castaño R, (1999). Medicina, Ética y reformas a la salud, "Hacia un Nuevo Contrato Social con la Profesión Médica, Eco Ediciones.

Castaño, G. (2001) Aportes a la economía de la empresa. Manizales: Universidad Nacional de Colombia.

Giles D.E., \& Draeseke R.(2001) Econometric Modelling Based on Pattem Recognition via the Fuzzy c-Means Clustering Algorithm, Department of Economics University of Victoria Working Paper.

LEY 100 DE 1993, (Diciembre 23), Diario Oficial No. 41.148, de 23 de diciembre de 1993. Ley 1122 de enero 9 de 2007.

Macho, I \& Pérez, C D. (1994) Introducción a la economía de la información: Barcelona Ed. Ariel S.A. 
Roach SS. (1991) Services under Sieger? The Restructuring Imperative. Harvard Business Review, September? October 1991, pp 82-91.

Spence M and Zeckhauser R, (1971) Insurance, Information, and Individual Action American Economic Association Pp 380-387.

Spence M, (2001). Signaling In Retrospect and the Informational Structure of Markets Príze Lecture, By A. Stanford Business School, Stanford University, 518 Memorial Way, Stanford, Ca 94305-5015, USA.

Spence M, Market SB; (1974) Quarterly Journal of Economic.

Stiglitz E AND Welss A., (1981) Credit Rationing in markets with imperfect Information; "The American Economic Review; PP 393, 410.

Stiglitz E., (2001) Information And The Change In The Paradigm In Economics Príze Lecture, December 8, Columbia Business School, Columbia University, 1022 International Affairs Building, 420 West 118th Street, New York, NY 10027, USA.

Várela, A, (2002). Asimetría de la información: Barreras para la implementación de la reforma de salud en Colombia. Colombia- Médica, Vol. 33, pp. 95,101.

Vera, A PhD. (2001) Microeconometrics and Asymmetric Information: "applications to health care utilization". Dissertation In economics: Barcelona Universidad Autónoma de Barcelona Belalterra. 


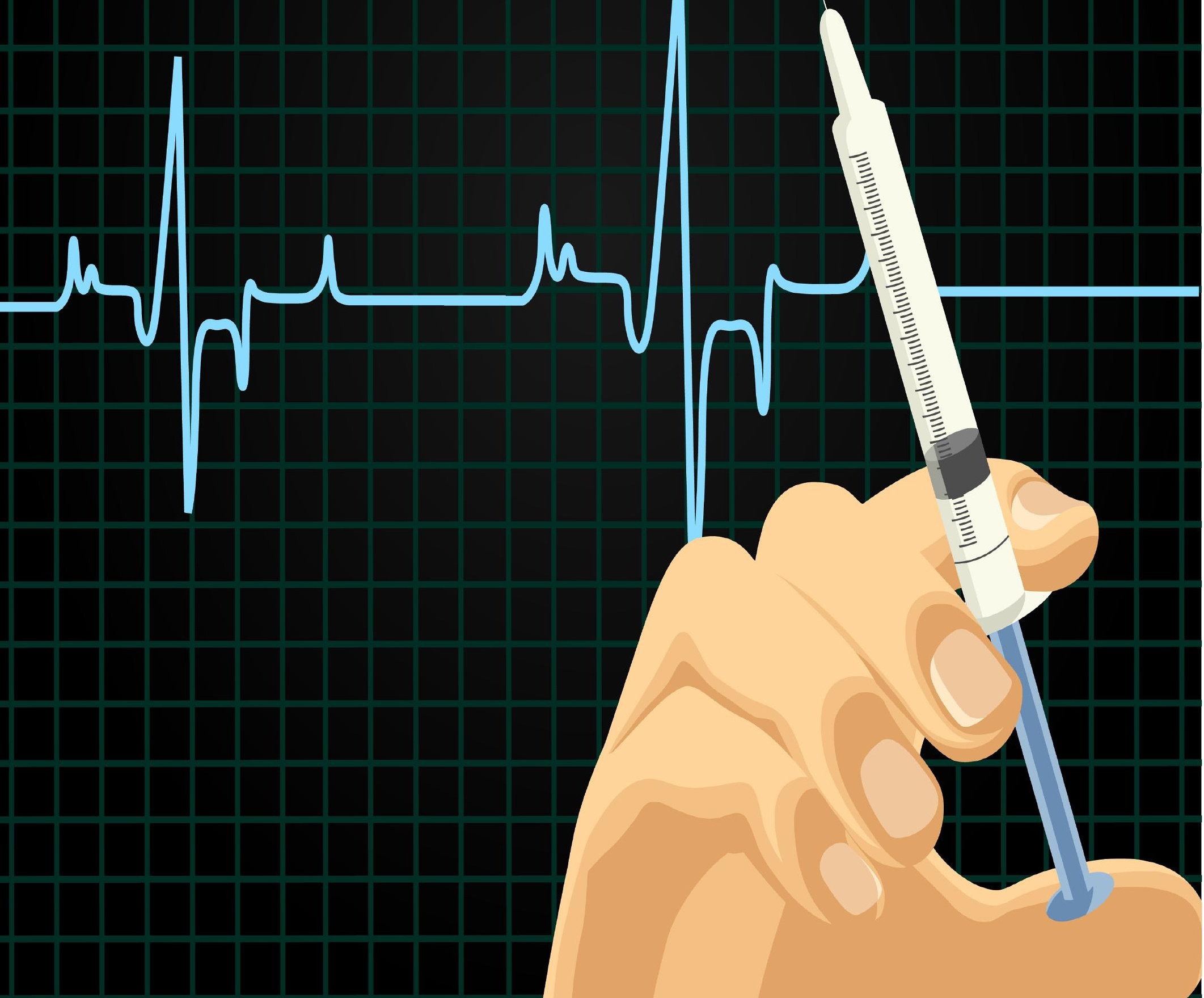

